\title{
Criação de banco de dados \\ para sustentação da pós-eliminação em hanseníase
}

\author{
Creation of a data bank \\ for sustainability of leprosy post elimination
}

\author{
Vânia Del Arco Paschoal ${ }^{1}$ \\ Susilene Maria Tonelli Nardi ${ }^{2}$ \\ M aria Rita de Cássia O liveira Cury ${ }^{1}$ \\ ClovisLombardi ${ }^{3}$ \\ M arcos da Cunha Lopes Virmond ${ }^{3}$ \\ Renée $M$ atar Dourado N eta da Silva ${ }^{1}$ \\ José Antonio Armani Paschoal ${ }^{4}$ \\ Lílian Carla M agalhais ${ }^{5}$ \\ Ellen Carolina M arques Conte ${ }^{1}$ \\ Rosina Maria M artins Kubota ${ }^{1}$ \\ Rosa M aria Cordeiro Soubhia ${ }^{1}$
}

${ }^{1}$ Faculdade de M edicina de São José do Rio Preto. R. Brigadeiro Faria Lima

5.416, Vila São Pedro.

15090-000 São José do Rio Preto SP.

vaniapaschoal@yahoo.com.br ${ }^{2}$ Divisão de Laboratório Regionais, Instituto Adolfo Lutz.

${ }^{3}$ Instituto Lauro de Souza

Lima.

${ }^{4}$ Armani Consult SC Ltda.

${ }^{5}$ Prefeitura M unicipal de Votuporanga.
Abstract São osédo Rio Preto reached theWorld $\mathrm{H}$ ealth Organization goal eliminating leprosy as a Public Health problem in the year 2006, with a prevalence of $0.79 / 10,000$ inhabitants. In order to warrant reliable information to promote management measures that keep this status, the aim of this study was to create a leprosy data bank in the city with information from 1998 to 2006. A data collection protocol wasused and it contained 74 clinical-epidemiological variables that were gathered from primary and secondary sources. This work was initiated with 442 cases regi stered in the municipal control program database, from those 168 were excluded and 85 were inserted. There were 24 cases not notified in the N ational Information System (SIN AN). The 74 variables collected will allow further studies about themes: epidemiological profiles, household contacts control, reactions and deficiencies, and others. The difficulties faced during the elaboration of the database were related to decoding medical files data and inconsistency in the SINAN. The frequent mistakes while entering the data weaken the information system and difficult the plan of precise actions regarding health. These facts are relevant to the quality of attention and the maintenance of the leprosy elimination status.

Key words Leprosy, Epidemiology, Elimination, Prevention and control, Organization and administration
Resumo São José do Rio Preto atingiu o parâme tro da O rganização M undial deSaúdede eliminação da hanseníase como problema de saúde pública em 2006, com prevalência de 0,79/10.000 habitantes. Para garantir informações fidedignas que permitam medidas gerenciais e manutenção deste quadro, o objetivo do estudo foi criar um banco de dados da hanseníase do município, no período de 1998 a 2006. U tilizou-se protocolo para coleta de dados de 74 variáveis clínico-epidemiológicas encontradas em fontes primárias e secundárias. Iniciou-se o trabalho com 442 casos existentes nas informações de dados do programa de controle do município; destes, 168 foram excluídose 85 inseridos; 24 não haviam sido notificados no Sistema de Informação de Agravos de Notificação (SINAN). As 74 variáveis coletadas possi bilitarão estudo posterior de temas, como o perfil epidemiológico, controle de comunicantes, reações, deficiências, entre outros. As dificuldades encontradas na elaboração do banco foram em relação à decodificação de dados dos prontuários e inconsistências nas anotações do SIN AN . Oserrosno registro dos dadosocorrem com frequência, debilitando informações e dificultando o planejamento das ações na área, fatores importantes para qualidade do atendimento e sustentação da eliminação da hanseníase.

Palavras-chave Hanseníase, Epidemiologia, Eliminação, Prevenção e controle, Organização e administração 


\section{Introdução}

São Josédo Rio Preto éum município situado no noroeste do Estado deSão Paulo epólo degrande desenvolvimento econômico. Centro urbano de dimensões médias, com crescentes atividades industriais, comerciais, agrícolas eculturais, éa sede da 8a região administrativa, englobando $101 \mathrm{mu}$ nicípios. Sua área total é de 434,10 quilômetros quadrados ${ }^{2}$, sendo a área urbana de 96,81 quilômetros quadrados, com uma população de 400.000 habitantes. Possui clima tropical e pluviosidade acentuada no período de outubro a março. A temperatura média é de $24,92^{\circ} \mathrm{C}$. Quanto às condições ambientais, tem indicadores próximos de $100 \%$ deatendimento deabastecimento deágua tratada, coleta de lixo e esgoto sanitário $0^{1,2}$.

A prevalência de hanseníase para São José do Rio Preto ésemelhantea do Estado de São Paulo no período de 1993 a 2003 2. Em 2002, contava com 45 casos novos de hanseníase, 79 pacientes em tratamento etaxa de prevalência de 2,1/10.000 habitantes. Em 2003, a taxa de prevalência foi de 1,49/10.00 habitantes, com 36 casos novos e 57 pacientes em tratamento. Em 2006, o município teve 41 casos em tratamento, com uma taxa de prevalência de $0,79 / 10.000$ habitantes, atingindo os parâmetros de eliminação preconizados pela O rganização M undial da Saúde (OM S). No ano de2007, o coeficientededetecção foi de $0,46 / 10.000$ habitantes ${ }^{3}$.

Com a implantação do Sistema de Informação de Agravos de N otificação (SINAN), nos últimos oito anos, a análise dos dados de um município tornou-se mais rápida e abrangente. Entretanto, este sistema apresenta al guns entraves, entre eles, as falhas de digitação 4,5 . Os dados são escritos manualmente, por vezes ininteligíveis, com várias cópias, incorrendo em erros. Além disto, são compilados de diversas fontes, como prontuários, fichas de cadastro da enfermagem e de controle de comunicantes, planilhas de inclusão, entre outras. Ademais, este acesso múltiplo aos dados dificulta a coleta de informações.

Para o controle da hanseníase, os gestores se deparam com várias dificuldades, tais como o desconhecimento por parte dos profissionais responsáveis pelas unidades de saúde, problemática dos pacientes dentro de sua área de abrangência, o treinamento de recursos humanos nos diferentes níveis profissionais, a adequada manutenção de estoques dos medicamentos específicos, o acesso geográfico e cultural dos próprios pacientes. Estes fatores, em conjunto com as características clínicoepidemiológicas da doença, levam à possibilidade do estabelecimento de uma prevalência oculta na comunidade, isto é, casos que são detectados somentemuito tardiamenteeque, antes disto, atuam como mantenedores edifusores da endemia. Estes são os casos que podem ser responsáveis por aumentos nas taxas de prevalência ${ }^{6}$ e podem ser estimados com vistas à previsão de atividades de controle por parte dos gestores. Esta estimativa, entretanto, dependeda precisão dosdadosdisponíveis ${ }^{7,8}$. De fato, para esta e outras tarefas gerenciais visando ao controle da endemia, o aprimoramento da qualidade dos dados gerando informações corre tas é fundamental para o bom desenvolvimento das ações e sustentação de uma situação epidemiológica de baixa preval ência e detecção.

\section{Objetivo}

Este trabalho teve como objetivo descrever a criação deum banco municipal de dados de hanseníase para estudo contínuo das variáveis clínicoepidemiológicas e sustentação da etapa de póseliminação no município deSão J osédo Rio Preto (SP).

\section{M etodologia}

O presente estudo foi aprovado pelo Comitê de Ética em Pesquisa da Faculdade de M edicina de São José do Rio Preto, Autarquia Estadual (FAM ERP), resolução CNS n 196/96, com o título "Em busca da pós-eliminação da hanseníase em São José do Rio Preto".

Trata-se de um censo descritivo, transversal, que teve início a partir da base de dados do SlNAN, disponibilizada pela Secretaria Municipal de Saúde e Higiene (SM SH) de São José do Rio Preto, Programa de Controle da $\mathrm{H}$ anseníase.

Os dados foram obtidos em duas unidades de saúde, o Ambulatório de Dermatologia do H ospital de Base (ADH B) e o Núcleo de Gestão Assistencial 60 (NGA-60), por meio dos registros encontrados em prontuários, nas fichas de controle de atendimento da enfermagem e nas fichas de notificações. Em São José do Rio Preto, 0 atendimento ainda é centralizado e somente estas unidades têm o programa de controle da hanseníase implantado no município.

0 instrumento de coleta de dados empregado foi uma ficha clínico-epidemiológica, quecontemplava o perfil demográfico dos pacientes, os aspectos clínicos, a situação do tratamento e do controle dos comunicantes intradomiciliares. 
A pós a coleta dos dados, eles foram introduzidos em planilha eletrônica, definindo-se as datas de inclusão e exclusão nos diagnósticos realizados, no período de 01/01/1998 a 30/12/2006. A definição desteperíodo decoleta ocorreu pelo fato de que os dados anteriores ao ano de 1998 foram considerados inconsistentes, já quevários pacientes tratados não estavam inseridos na base de dados do SINAN ou da SM SH, e os que estavam, apresentavam dados incompletos. Possivelmente, o exercício da construção dos bancos pode ter sido um dos fatores que contribuiu para a melhoria na consistência dos dados ao longo dos anos.

Para a realização do estudo, isto é, a criação de um banco de dados, nomeado de Banco de Dados do Projeto Hansen, os encontros de planejamento, coordenação, estudos e informações ocorreram naFaculdade de M edicina de São J osé do Rio Preto (FAM ERP).

Participaram da coleta e organização dos dadosoito docentes, trinta alunos do curso demedicina eenfermagem, dois pesquisadores do Instituto Lauro de Souza Lima (ILSL), a interlocutora da hanseníase, dois funcionários da Secretaria Municipal de Saúde e Higiene de São José do Rio Preto e três voluntários no transporte de alunos para o ADH B eo NGA-60. Os participantes trabaIharam em mutirão ou individualmente três dias por semana, no período de 17/06/06 a 31/12/06.

\section{Resultados}

Limpeza dos dados,

reconhecimento dos doentes reais

A base de dados do SINAN no período de 1999 a 2005 apresentava 553 doentes notificados. 0 estudo realizado por Curto et al. ${ }^{9}$ descreveu os resultados parciais da criação do banco, levando o grupo de pesquisadores a redefinir o período do estudo e os critérios de inclusão e exclusão para a 01/01/1998 a 31/12/2006 einserir no Banco de Dados do Projeto H ansen apenas moradores do município. Iniciou-se, então, a coleta de dados, com informação de 442 doentes incluídos na base de dados do SINAN e, após a finalização da coleta e análise, 85 pacientes foram incluídos, 168 excluídos, resultando em 359 casos dehanseníase no período já descrito. Os motivos da inclusão foram os casos não notificados no sistema de informação oficial $(n=24)$ ea entrada de novos do período acrescentado $(n=61)$. Os excluídos foram casos tratados em datas anteriores ao período estipulado no estudo e os que iniciaram tratamento no município e depois foram transferidos para outras cidades. Foram também retirados da base de dados os pacientes deoutros municípios notificados em São José do Rio Preto, os óbitos, as mudanças de diagnóstico que ainda permaneciam e as notificações oficiais duplicadas ( $n=168$ ).

Foram escolhidos onzetemas principais: identificação do cliente, endereço oficial, dadosem relação à hanseníase, data e resultado da avaliação de prevenção de incapacidades, tratamento realizado, estados reacionais, neurites, datas deacompanhamento, outros medicamentos utilizados, identificação de fonte de transmissão e os comunicantes intradomiciliares. Deste modo, foram geradas 74 variáveis, ou 26.566 áreas de informações, distribuídas por assunto (Quadro 1).

Vantagens e dificuldades encontradas durante o processo de coleta dos dados

\section{Em relação ao SI NAN}

A centralização dos dados dificulta não só a realimentação para os funcionários que colaboram no repasse das informações, como também a análise destes dados demaneira conjunta pelos atores envolvidos nesta construção. A atualização incompleta e por vezes incorreta de dados importantes, como estados reacionais, não é computada. D escobriu-se a subnotificação de 24 pacientes. Acredita-se que este fato tenha ocorrido provavelmente pelo fato do SINAN não estar até então na WEB e pelo grande número de pessoas que manipularam estes dados, desde o preenchimento da ficha de notificação no momento do diagnóstico atéa digitação no sistema da Vigilância Epidemiológica. Pelo longo trajeto que estes dados percorreram, estão propensos a desvios, negligências, erro de digitação e perdas. Um exemplo foi 0 encontro de fichas de notificação preenchidas dentro dos prontuários sem notificação oficial.

No que tange ao presente estudo, partes dessas dificuldades foram sanadas por se contar previamente com um banco já instituído no município, que agiu como uma memória para a criação do banco objeto deste estudo (Banco de Dados do Projeto $\mathrm{H}$ ansen) e adequação da base de dados do SINAN.

Em relação às unidades de controle

Não há metodologia estabelecida e padronizada para as anotações feitas pelos profissionais nos prontuários, dificultando a análise dos fatos ocorridos durante a doença e a comparação entre os dois serviços. 
Quadro 1. I dentificação e caracterização das variáveis obti das das diversas fontes de coleta de dados.

\begin{tabular}{|c|c|c|c|c|}
\hline Fonte de coleta & \multicolumn{4}{|c|}{ Variável } \\
\hline \multirow{13}{*}{$\begin{array}{l}\text { Prontuário } \\
\text { SINAN } \\
\text { Ficha de } \\
\text { controle de } \\
\text { enfermagem }\end{array}$} & \multirow{8}{*}{$\begin{array}{l}\text { Identificação do } \\
\text { cliente }\end{array}$} & \multirow[t]{3}{*}{ No serviço } & № do prontuário & - \\
\hline & & & Código do SINAN & - \\
\hline & & & Local de tratamento & AHB ou NGA-60 \\
\hline & & Nome do paciente & - & - \\
\hline & & \multirow[t]{3}{*}{ Data nascimento } & Dia & \multirow[t]{3}{*}{-} \\
\hline & & & M ês & \\
\hline & & & Ano & \\
\hline & & Gênero & Masculino ou feminino & \\
\hline & \multirow{5}{*}{ Endereço oficial } & Rua & - & - \\
\hline & & Número & - & \\
\hline & & Bairro & - & \\
\hline & & CEP & - & \\
\hline & & Telefone & $\begin{array}{l}\text { Residencial, } \\
\text { celular, } \\
\text { profissional } \\
\text { de contato }\end{array}$ & - \\
\hline \multirow{15}{*}{$\begin{array}{l}\text { Prontuário } \\
\text { Resultados de } \\
\text { exames }\end{array}$} & \multirow{15}{*}{$\begin{array}{l}\text { Em relação ao } \\
\text { diagnóstico eà } \\
\text { hanseníase }\end{array}$} & \multirow[t]{8}{*}{ Classificação clínica } & Lesão única & \\
\hline & & & $\mathrm{MHI}$ & \\
\hline & & & MHT & \\
\hline & & & MHDT & \\
\hline & & & MHDD & \\
\hline & & & MHDV & \\
\hline & & & MHV & \\
\hline & & & Neural pura & \\
\hline & & \multirow{3}{*}{$\begin{array}{l}\text { Teste de baciloscopia } \\
\text { (BAAR) }\end{array}$} & Positivo & Número decruzes \\
\hline & & & Negativo & - \\
\hline & & & Não realizado & - \\
\hline & & \multirow[t]{3}{*}{ Teste de M itsuda } & Positivo & $\mathrm{mm}$ do endurecimento \\
\hline & & & Negativo & - \\
\hline & & & Não realizado & - \\
\hline & & Examehistopatológico & $\begin{array}{c}\text { MHI, MHT, MHDT, } \\
\text { MHD, MHDV, MHV } \\
\text { Não realizado }\end{array}$ & - \\
\hline \multirow{11}{*}{$\begin{array}{l}\text { Prontuário } \\
\text { Ficha de } \\
\text { controle de } \\
\text { enfermagem } \\
\text { Ficha de } \\
\text { avaliação de } \\
\text { incapacidades }\end{array}$} & \multirow{11}{*}{$\begin{array}{l}\text { Prevenção de } \\
\text { incapacidades }\end{array}$} & \multirow{3}{*}{$\begin{array}{l}\text { Data da avaliação no } \\
\text { diagnóstico }\end{array}$} & Olhos & \multirow{3}{*}{ Direito eesquerdo } \\
\hline & & & Mãos & \\
\hline & & & Pés & \\
\hline & & Sem data & - & \\
\hline & & \multirow{3}{*}{$\begin{array}{l}\text { Data da avaliação na } \\
\text { alta }\end{array}$} & Olhos & \multirow{3}{*}{ Direito e esquerdo } \\
\hline & & & Mãos & \\
\hline & & & Pés & \\
\hline & & Sem data & - & - \\
\hline & & $\mathrm{EHF}$ & Grau máximo & - \\
\hline & & OMS & Grau M aximo & - \\
\hline & & Avaliador & Nome & - \\
\hline \multirow{4}{*}{$\begin{array}{l}\text { Prontuário } \\
\text { Ficha de } \\
\text { controle de } \\
\text { enfermagem }\end{array}$} & \multirow{4}{*}{$\begin{array}{l}\text { Tratamento } \\
\text { realizado }\end{array}$} & PQT & $\begin{array}{l}\text { PB } \\
\text { M B }\end{array}$ & - \\
\hline & & DNDS & - & \\
\hline & & Outro & Alternativo & - \\
\hline & & $\mathrm{N}$ úmero de doses PQT & M eses & - \\
\hline
\end{tabular}


Quadro 1. continuação

\begin{tabular}{|c|c|c|c|c|}
\hline Fonte de coleta & \multicolumn{4}{|c|}{ Variável } \\
\hline \multirow{44}{*}{$\begin{array}{l}\text { Prontuário } \\
\text { Ficha de } \\
\text { controle de } \\
\text { enfermagem }\end{array}$} & \multirow{13}{*}{$\begin{array}{l}\text { Tratamento } \\
\text { realizado }\end{array}$} & $\begin{array}{l}N \text { úmero de } \\
\text { atendimento }\end{array}$ & $\begin{array}{l}\text { PQT } \\
\text { Outras }\end{array}$ & - \\
\hline & & \multirow{5}{*}{ Tipo deprofissional } & M édico & \\
\hline & & & Enfermeira & \\
\hline & & & Terapeuta Ocupacional & \\
\hline & & & Fisioterapeuta & \\
\hline & & & Psicólogo & \\
\hline & & \multirow[t]{2}{*}{ Falta-doses } & Sim & \multirow[t]{2}{*}{ Quantas } \\
\hline & & & Não & \\
\hline & & \multirow{2}{*}{$\begin{array}{l}\text { Efeitos colaterais } \\
\text { drogas da PQT }\end{array}$} & Sim & \multirow[t]{2}{*}{ Quais } \\
\hline & & & Não & \\
\hline & & \multirow[t]{3}{*}{ Situação atualizada } & Em tratamento & - \\
\hline & & & Em alta & \\
\hline & & & Abandono & \\
\hline & \multirow[t]{13}{*}{$\begin{array}{c}\text { Estadosreacionais } \\
\text { Neurites }\end{array}$} & Sim & ENH, RR & $\begin{array}{c}\text { Neurites } \\
\text { Fenômeno de Lucio, } \\
\text { Orbaneja }\end{array}$ \\
\hline & & Não & - & - \\
\hline & & $\begin{array}{c}\text { Auricular maior, facial, } \\
\text { trigêmeo }\end{array}$ & Direito & \multirow{3}{*}{-} \\
\hline & & Ulnar, mediano, radial & Esquerdo & \\
\hline & & Tibial posterior, fibular & & \\
\hline & & \multirow{2}{*}{ Reações ou neurite } & Antes do tratamento & \multirow[b]{2}{*}{-} \\
\hline & & & $\begin{array}{c}\text { No tratamento } \\
\text { Após o tratamento }\end{array}$ & \\
\hline & & Tempo de reações & Episódios de reações & - \\
\hline & & Tempo de neurite & Episódios deneurite & - \\
\hline & & $\begin{array}{c}\text { M edicamentos } \\
\text { utilizados }\end{array}$ & $\begin{array}{l}\text { Sim } \\
\text { Não }\end{array}$ & - \\
\hline & & Neuropatia crônica & & - \\
\hline & & M edicamentosusados & Nome & - \\
\hline & & Assintomático & Meses & - \\
\hline & \multirow{5}{*}{$\begin{array}{c}\text { Datas de } \\
\text { acompanhamento }\end{array}$} & Início dos sintomas & \multirow{5}{*}{$\begin{array}{l}\text { Dia } \\
\text { M ês } \\
\text { Ano }\end{array}$} & \\
\hline & & Notificação & & \\
\hline & & Início do diagnóstico & & \\
\hline & & Início do tratamento & & \\
\hline & & Alta quimioterápica & & \\
\hline & \multirow{2}{*}{$\begin{array}{c}\text { Outros } \\
\text { medicamentos } \\
\text { utilizados }\end{array}$} & Sim & \multirow[t]{2}{*}{ Quais } & \multirow[t]{2}{*}{-} \\
\hline & & Não & & \\
\hline & \multirow{3}{*}{$\begin{array}{l}\text { Identificação de } \\
\text { fonte de } \\
\text { transmissão }\end{array}$} & Sim & Consanguíneo & - \\
\hline & & Não & Não consanguíneo & - \\
\hline & & Não informado & & \\
\hline & \multirow{8}{*}{ Comunicantes } & Número & Nome & \\
\hline & & \multirow[t]{3}{*}{ Grau de parentesco: } & Esposos/ pais & \\
\hline & & & Filho, irmão, neto, sobrinho & \\
\hline & & & Irmão, etc. & \\
\hline & & \multirow[t]{3}{*}{ Avaliados } & \multirow[t]{3}{*}{ Número de vezes } & Em visita ao programa \\
\hline & & & & Em visita domiciliar \\
\hline & & & & Em outro serviço \\
\hline & & BCG & $\begin{array}{l}0 \text { (nenhuma dose) } \\
1 \text { ( uma dose) } \\
2 \text { (duas doses) }\end{array}$ & - \\
\hline
\end{tabular}


N o ADHB, os prontuários são guardados no Serviço de Arquivo M édico Estatístico (SAME), junto de todos os prontuários do complexo hospitalar. Sua solicitação é feita somente mediante agendamento prévio, o que pode demorar até dois dias. Os prontuários tornam-seinativos após cinco anos de desuso e são encaminhados para depósito, dificultando ainda mais o acesso às pesquisas. Em contrapartida, esses prontuários são utilizados por todas as especialidades e retratam de forma multidisciplinar a evolução clínica do doente na instituição, sendo uma vantagem para a clínica e desvantagem para a coleta de dados e pesquisa.

N o NGA-60, ao contrário, os prontuários só retratam a evolução da hanseníase, o que favorece e minimiza o tempo gasto na extração de dados para pesquisa, mas dificulta a visão geral da evolução clínica e laboratorial dos doentes, para a atuação clínica.

\section{Em relação aos prontuários}

As anotações em prontuários e nas fichas de controle da enfermagem ora se encontravam organizadas e sistematizadas, ora estavam esparsas ou incompletas e em diferentes locais dentro da unidade de saúde (caderno de anotações do funcionário, fol has avulsas dentro de pastas) dificultando 0 acesso às informações. As anotações dos atendimentos de consultas são colocadas em sequência única por todas as especialidades, como médico, enfermagem, terapeuta ocu-

Tabela 1. Relação das inconsistências encontradas entre o banco inicial com o Banco de Dados do Projeto Hansen.

\begin{tabular}{|c|c|c|c|c|}
\hline \multirow{3}{*}{ Item } & \multicolumn{4}{|c|}{ Informação } \\
\hline & \multicolumn{2}{|c|}{ Incorreta } & \multicolumn{2}{|c|}{ Inexistente } \\
\hline & $N$ & $\%$ & $\mathbf{N}$ & $\%$ \\
\hline Nome & 18 & 5,01 & - & - \\
\hline Rua & 50 & 13,90 & 1 & 0,28 \\
\hline $\mathrm{N}^{\circ}$ da residência & 40 & 11,14 & 6 & 1,67 \\
\hline Bairro & 12 & 3,34 & 205 & 57,10 \\
\hline CEP & 3 & 0,84 & 222 & 61,84 \\
\hline Telefone & 63 & 17,55 & 99 & 27,58 \\
\hline Data de nascimento & 11 & 3,06 & 0 & - \\
\hline Forma clínica & 28 & 7,80 & 2 & 0,56 \\
\hline Grau de incapacidade no diagnóstico & 62 & 17,27 & 56 & 15,60 \\
\hline $\begin{array}{l}\text { Comunicantes que residiam com o } \\
\text { doente em até } 5 \text { anos antes do } \\
\text { diagnóstico }\end{array}$ & 137 & 38,16 & 13 & 3,62 \\
\hline
\end{tabular}

$n=359$. pacional efisioterapeuta. No ADHB, também ficam junto com todas as outras especialidades, inclusive os exames de laboratório, dificultando, desta forma, a coleta de dados e a visão do estado do pacienteespecificamenteem relação àhanseníase. Os prontuários trazem os exames, consultas e anotações muitas vezes sem sequência lógica das datas de atendimento. Outros agravantes são a letra ilegível, fal ta de data eassinatura do profissional que realizou 0 atendimento.

N os prontuários do N GA-60 não estão estabelecidas como rotina, a atualização de endereço, a referência a óbitos e as informações sobre comunicantes, entre outras ocorrências, mesmo que o paciente seja assíduo ao serviço de saúde. Como sevê, não há no município um protocolo específico para 0 atendimento em hanseníase, como ocorre, pelo menos para a enfermagem, nos atendimentos básicos de saúde.

Para se ter uma idéia das disparidades dos dados encontrados nos prontuários em relação às demais fontes, na Tabela 1 se descrevem algumas das inconsistências encontradas e corrigidas pela equipe do estudo.

\section{Em relação ao Banco de Dados do Projeto Hansen}

Como fato de maior relevância, a criação do banco de dados ofereceu a oportunidade de se conhecer, com maior precisão, o número de pessoas que estão em tratamento ou já tratou a hanseníase em cada pólo regional (Figura 1 e Tabela 2) e, muito importante, facilitar o controle de seus comunicantes por parte das unidades básicas de saúde. Isto, em última análise, se traduz por uso adequado de ferramenta gerencial para a efetiva condução das ações de controle da endemia. Os dados, subsidiariamente, ainda permitem a construção de mapas geoprocessados, para a visual ização da distribuição espacial etemporal dos casos, como mostra a Figura 1.

A maior concentração de casos está na região nortedo município; destes, nove pessoas permaneceram fora da área de abrangência, pois no endereço coletado não havia sintonia com as informações existentes, ora eram moradores da zona rural, ora os dados estavam incompletos, ora viviam em áreas não oficialmentelegalizadas do município.

A distribuição espacial dos casos dehanseníase no município nos anos de 1998 a 2006 em São José do Rio Preto é apresentada na Figura 1. 


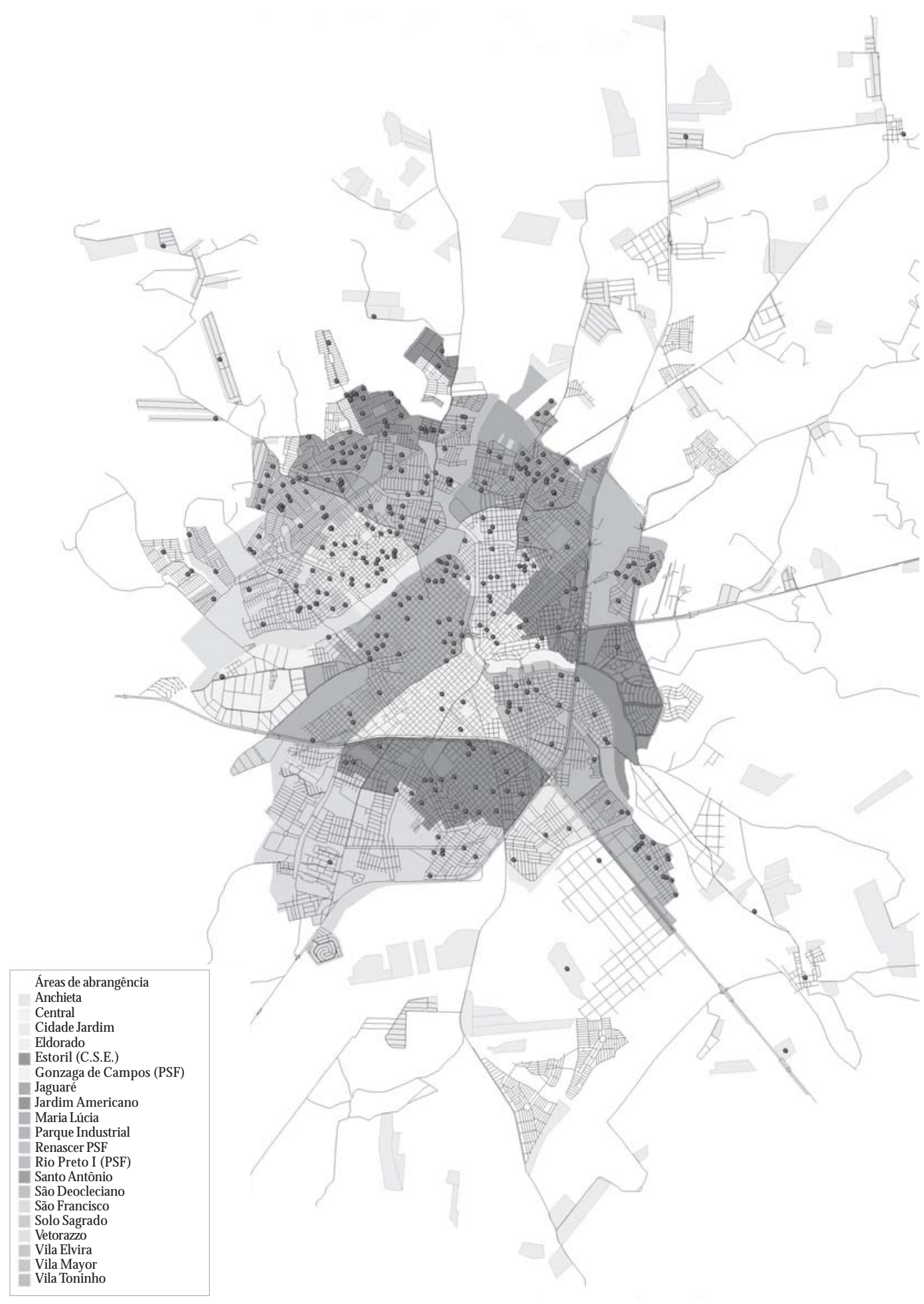

Figura 1. Distribuição espacial dos casos de hanseníase nos anos de 1998 a 2006. São José do Rio Preto, 2007. 


\begin{tabular}{|c|c|c|c|c|c|c|}
\hline \multirow[t]{8}{*}{ 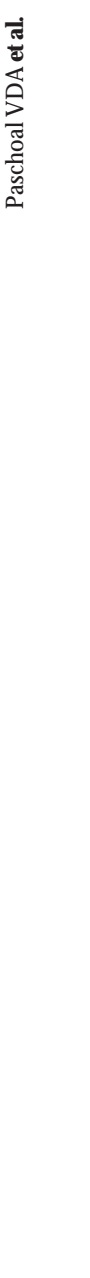 } & $\begin{array}{l}\text { Tabela } \\
\text { área de }\end{array}$ & $\begin{array}{l}\text { ação dos casos } \\
\text { igência. São Jc }\end{array}$ & $\begin{array}{l}\text { anseníase, } \\
\text { Rio Pretc }\end{array}$ & ulação, bairro & domicílios e & nidades Básicas de Saúde da \\
\hline & Polo & $\begin{array}{c}\text { Casos de } \\
\text { hanseníase }\end{array}$ & Bairros & População & Domicílios & UBS \\
\hline & 1 & 90 & 160 & 126.567 & 45.495 & $\begin{array}{l}\text { Amb. Esp. NGA-60; } \\
\text { UBS Central; } \\
\text { UBS Vila Elvira; } \\
\text { UBS Jardim Americano; } \\
\text { UBS Pq Industrial; } \\
\text { UBS Anchieta }\end{array}$ \\
\hline & II & 152 & 84 & 121.164 & 33.120 & $\begin{array}{l}\text { Policlínica Sto. Antônio; } \\
\text { Policlínica Ipê Branco; } \\
\text { Policlínica Vetorasso; } \\
\text { UBSF Pq. da Cidadania; } \\
\text { UBSF Renascer; } \\
\text { UBSF Gonzaga de Campos; } \\
\text { UBS Vila M ayor; } \\
\text { UBS Eldorado }\end{array}$ \\
\hline & III & 33 & 54 & 57.673 & 16.003 & $\begin{array}{l}\text { CSEscola do Pq. Estoril; } \\
\text { UBSaúde São Francisco }\end{array}$ \\
\hline & IV & 26 & 23 & 25.207 & 8.249 & $\begin{array}{l}\text { Policlínica Vila Toninho; } \\
\text { UBSF Cidade Jardim; } \\
\text { UBS Engenheiro Schimidt }\end{array}$ \\
\hline & V & 49 & - & 46.932 & - & $\begin{array}{l}\text { Policlínica Jaguaré; } \\
\text { UBS S. Deocleciano; } \\
\text { UBSF Talhado; } \\
\text { UBSF Rd. Rio Preto }\end{array}$ \\
\hline & Total & 350 & 321 & 377.543 & 102.867 & \\
\hline
\end{tabular}

\section{Discussão}

Com vista a monitorar epidemiologicamente as doenças, em 1993, o M inistério da Saúde criou o Sistema de I nformações de Agravos de N otificação (SINAN), que condensa dados sobre morbidade. A pós catorzeanos da sua criação, profissionais de saúde encontram inconsistências que ge ram dificuldades no planejamento de ações de controle, seja no município, estado ou país. Adicionalmente, o SINAN deveria auxiliar no monitoramento da meta de eliminação da hanseníase como problema de saúde pública, conforme os critérios acordados entre o M inistério da Saúde e a Organização M undial da Saúde.

Entretanto, o fluxo interno dos impressos, a falta de registro no momento do diagnóstico, os erros de digitação e o repasse de informação de um serviço para outro podem justificar as inconsistências encontradas. De fato, estudo realizado no Ceará detectou subnotificação de 14,9\% de casos de hanseníase se comparado com os dados disponíveis no nível local ${ }^{5}$. Portanto, seja para planejamento de ações ou para subsidiar pesquisas, há urgência na obtenção de consistência e consequente confiabilidade nos dados registrados em um sistema de informações de dados. Ademais, para se fortalecer a vigilância dos casos, os índices epidemiológicos devem ser estimados o mais próximo possível da realidade de cada local ou região, para estabelecimento de metas adequadas de eliminação ou pós-eliminação pelos países endêmicos ${ }^{10}$. A atualização dos dados requer motivação de funcionários operacionais, digitadores e gestores, no sentido da obtenção e análise dos índices locais e regionais. Descobrir distorções e corrigi-las possibilita me Ihor seguimento eavaliação do programa decontrole, tornando-o menos vulnerável ${ }^{11,12}$. Uma possível forma de atingir tal objetivo seria simplificar os atuais métodos de coleta e análise dos dados, melhorando sua qualidade ${ }^{13}$. Uma outra possibilidade, como foi observado no presente estudo, équeas reavaliações periódicas para correção e atualização dos dados podem gerar fidelidade das informações dos sistemas conhecidos. 
Outro fator adicional, mas não menos importante, a ser considerado na criação de bancos de dados é que, quando se observa a hanseníase como doença infecciosa, ela termina na alta do tratamento poliquimioterápico, mas quando são considerados os aspectos de incapacidade e de transmissão, ela se torna mais complexa, abrangente e o cuidado deve ser mais amplo. Este aspecto também é relevante quando a área se encontra na etapa de baixa prevalência e há necessidadedeconsiderar osíndices epidemiológicospara avaliação constante das ten dências de evolução e variações, permitindo intervenções ágeis efocais ${ }^{14}$.

\section{Considerações finais}

A criação do Banco de Dados do Projeto $\mathrm{H}$ ansen ofereceu ao município de São José do Rio Preto (SP) dados atualizados, disponíveis aos profissionais da área da saúde, colaborando para atenção ao paciente, controle da endemia e pesquisa.

A distribuição dos pacientes dehan seníase por pólos regionais oferece maior facilidade de acesso às informações, possibilitando o controle mais efetivo dos pacientes e comunicantes pelos profissionais das unidades de saúde e ou programas de atenção básica, como o de saúde da família.

\section{Colaboradores}

VDA Paschoal, SMT Nardi eRM C Soubhia coordenaram a pesquisa e trabalharam em todas as etapas da mesma; M RCO Cury trabalhou nas etapas de planejamento e levantamento de dados; C Lombardi e M CL Virmond assessoraram a pesquisa e trabal haram nas etapas de planejamento eredação do texto; RM DN Silva, JAA Paschoal, ECM Conte e RM M Kubota trabalharam nas etapas de planejamento e levantamento de dados e LC Magalhais trabalhou nas etapas de planejamento, levantamento de dados e redação do texto.

\section{Agradecimentos}

Esta pesquisa teve o apoio financeiro do CN PqEdital MCT-CNPq/ MS-SCTIE-DECIT no. 35/ 2005. 


\section{Referências}

1. Prefeitura de São José do Rio Preto. Secretaria Municipal de Planejamento de Gestão Estratégica. Conjuntura Econômica.19a ed. São José do Rio Preto: Secretaria M unicipal de Planejamento de Gestão Estratégica; 2004.

2. Chiaravalloti Neto F. A Hanseníase em São José do Rio Preto. Bol Epidemiol 2004; 7.

3. Brasil. Ministério da Saúde. DATASUS. [site da Internet] [acessado 2007 set 12]. Disponível em: http:/ /tabnet.datasus.gov.br/cgi/tabcgi.exe?hans/hanswsp. def

4. Nardi SMT, Marciano LHSC, Virmond MCL, Baccarelli R. Sistemas de informação e deficiências físicas na hanseníase. BEPA 2006; 3(27):3-7.

5. Façanha MC, Pinheiro AC, Lima JRC, Ferreira MLLT, Teixeira GFD, Rouquayrol MZ. Hanseníase: subnotificação de casos em Fortaleza, Ceará, Brasil. An Bras Dermatol 2006; 81(4):329-333.

6. Cunha MD, Cavaliere FAM, Hércules FM, Duraes SM B, Oliveira M LW, M atos, HJ. Os indicadores da hanseníase e as estratégias de eliminação da doença, em município endêmico do Estado do Rio de Janeiro, Brasil. Cad Saude Publica 2007; 23(5):1187-1197.

7. Suárez REG, Lombardi C. Estimado de prevalência de lepra. Hansen Int 1997; 22(2):31-34.

8. Ferreira J, Mengue SS, Wagner M B, Duncan BB. Estimating hidden prevalence in $\mathrm{H}$ ansen's disease through diagnosis delay and grade of disability at time of diagnosis. Int J Lepr 2000; 68(4):464-473.

9. Curto M, Paschoal VDA. Uma década de acompanhamento dos portadores de hanseníase no ambulatório de dermatologia de um hospital-escola. Arq Ciênc Saúde 2005; 12(4):183-195.

10. Organização Mundial da Saúde. Estratégia global para aliviar a carga da hanseníase e manter as atividades de controle, período do plano de 2006- 2010. [site da Internet] [acessado 2007 mai 10]. Disponível em: http://www.opas.org.br/prevencao/site/ U ploadArq/Estratégia_Global.pdf

11. Lombardi C, organizador. Hanseníase: epidemiologia e controle. São Paulo: Impressa Oficial do Estado; 1999.

World Health Organization. Anonymous. Guide to

12. eliminate leprosy. Geneva: WHO; 2000.

Andrade VLG. Características epidemiológicas da

13. hanseníase em área urbana: município de São Gonçalo [dissertação]. Rio de Janeiro (RJ): Escola Nacional de Saúde Pública Sérgio Arouca, Fundação Oswaldo Cruz; 1990.

Virmond MCL. Hansen's disease as a low preva-

14. Ience disease. Hansen. Int 1995; 20(2):36-45.

Artigo apresentado em 15/08/2008

Aprovado em 29/01/2009

Versão final apresentada em 21/02/2009 